\title{
Long noncoding RNA BLACAT1 promotes cell proliferation and invasion in human cervical cancer
}

\author{
DAN SHAN, YUMIN SHANG and TONGXIU HU
}

Department of Gynaecology and Obstetrics, Tianjin Hospital, Tianjin 300211, P.R. China

Received April 11, 2017; Accepted October 25, 2017

DOI: $10.3892 / 01.2018 .7773$

\begin{abstract}
Cervical cancer is one of the leading causes of mortality in females worldwide. Predisposition to distant metastasis has reduced the prognosis of this malignancy, thus the identification of a novel agent for metastatic cervical cancer is required. Long noncoding RNAs (LncRNAs) have been reported to serve significant roles in human tumorigenesis. The present study aimed to investigate the effects of a newly discovered LncRNA bladder cancer associated transcript 1 (non-protein coding) (BLACAT1) on cell proliferation and metastasis in cervical cancer. A total of 100 patients with cervical cancer were included, and tumor tissues as well as the adjacent non-cancerous counterparts were collected for reverse transcription-quantitative polymerase chain reaction analysis. It was demonstrated that BLACAT1 was highly expressed in human cervical cancer tissues and cell lines. The knockdown of BLACAT1 with specific short hairpin RNA reduced colony formation rates in ME180 and C33A cells. Cell cycle and cell proliferation assays revealed that depletion of BLACAT1 in ME180, and C33A cells arrested the cell cycle at the G0/G1 phase and inhibited cell proliferation. Transwell assays demonstrated that the knockdown of BLACAT1 inhibited cell migration and invasion in ME180, and C33A cells. Moreover, wound-healing assays supported the aformentioned observations. Western blot analysis showed that the knockdown of BLACAT1 in ME180 and C33A cells decreased the protein levels of cyclin B1, cell division cycle $25 \mathrm{C}$, and $\mathrm{N}$-cadherin, while increasing the protein level of E-cadherin. These findings indicated the oncogenic potential of BLACAT1 in cervical cancer, which may provide novel insights for the clinical diagnosis and treatment of cervical cancer.
\end{abstract}

Correspondence to: Dr Tongxiu Hu, Department of Gynaecology and Obstetrics, Tianjin Hospital, 406 South Jiefang Road, Hexi, Tianjin 300211, P.R. China

E-mail: tongxiu1234hu@yeah.net

Key words: long noncoding RNA, BLACAT1, proliferation, metastasis, cervical cancer

\section{Introduction}

Cervical cancer is one of the most common gynecological malignancies. Based on a recent statistic, more than 520,000 cases are newly reported annually (1). Traditional treatments for cervical cancer consist of surgery, chemotherapy and radiotherapy. Despite great advances in surgery and chemo-radiotherapy for the last decades, the long-term prognosis for patients suffering from cervical cancer remains still poor with a 5-year survival rate of less than $30 \%$ (2). The poor outcome of cervical cancer patients is largely due to the property of metastasis or recurrence (3). In fact, it has been well documented that a common feature of cervical cancer is an uncontrolled cell growth and metastasis (4). Thus, it is mandatory to find novel markers for the early diagnosis and treatment of cervical cancer.

With the rapid development of genome and transcriptome sequencing technologies and implement of genomics consortiums such as ENCODE and FANTOM, the classic view of the transcriptome landscape and its mRNA-centric paradigm for transcript annotation has undergone a fundamental change $(5,6)$. It has now been well established that more than $90 \%$ of the genome can be transcribed with only $<2 \%$ being subsequently translated. This means that the vast majority of genome serves as the template for the transcription of noncoding RNAs (ncRNAs) (7).

Long noncoding RNAs (lncRNAs) are a newly emerged class of noncoding RNA containing more than 200 nucleotides that are widely transcribed in the genome. Unlike other noncoding RNAs such as microRNAs, IncRNAs involvement in human diseases remains largely uncovered. Current knowledge has implicated that IncRNAs may widely participate in multiple intracellular and extracellular activities, including gene transcription, mRNA splice and tumorigenesis $(8,9)$. Emerging evidence have assessed the functional roles of specific lncRNA in cervical cancer so far. For instance, the lncRNA TUG1 was found to be upregulated and promoted cervical cancer proliferation and migration (10). Expression quantitative trait loci in lncRNA PAX8-AS1 are associated with decreased risk of cervical cancer (11). LncRNA CCAT2 promoted the proliferation and survival of cervical cancer (12).

BLACAT1 (also known as linc-UBC1) is one of the few well-known lincRNAs (one class of lncRNAs), with a length of 2,616 bp. BLACAT1 was firstly characterized in bladder cancers and exerts a functional role in recruiting and binding 
to polycomb repressive complex 2 (PRC2) (13). Recent studies have suggested that BLACAT1 exhibited tumor pro-oncogenic activity in gastric cancer (14), and may also serve as a negative predictor for prognosis in patients with gastric cancer (14) and colorectal cancer (15). These pioneer studies implied that BLACAT1 might be functional in solid tumor. However, whether BLACAT1 has any functional role in cervical cancer remains to be elucidated.

The present study sought to examine the expression profile of BLACAT1 in cervical cancer and then assess the biological roles of BLACAT1 in cervical cancer. Our results showed that BLACAT1 was upregulated in cervical cancer and its upregulation remarkably promoted cell proliferation and migration. Our data might provide novel evidence for development of therapeutic strategies against cervical cancer in clinic.

\section{Materials and methods}

Human samples. The present study was approved by the Ethic Committee of Tianjin Hospital. Cervical cancer tissues from 100 patients (age range, 45-72 years; mean age, 55 years; male: Female $=63: 37$ ) admitted to the Department of Gynaecology and Obstetrics, Tianjin Hospital between April 2015 and May 2016 were collected via surgical resection and frozen into liquid nitrogen immediately and then stored at $-80^{\circ} \mathrm{C}$. Matched adjacent non-cancerous tissues were also obtained. All patients showed their full intentions to participate in the present study and a written consent form was obtained from each patient.

Cell culture and transfection. Primary normal cervical epithelial cells (NCECs) were from non-cancerous cervical tissues, and were purchased from American Type Tissue Collection (ATCC, Massachusetts, USA). Cervical cancer cell lines Siha, CaSki, Hela, ME180 and C33A were commercially from the Cell Bank of Chinese Academy of Sciences (Shanghai, China). All of the cell lines were cultured in DMEM (Gibco, Grand Island, NY, USA) supplied with $10 \%$ fetal bovine serum (FBS; Gibco) and $1 \%$ antibiotics (penicillin/streptomycin). The cells were incubated in a $37^{\circ} \mathrm{C}$ incubator with $5 \% \mathrm{CO}_{2}$. Specific shRNA against BLACAT1 (shBLACAT1) and control shRNA (shNC) were designed and synthesized by Genepharm Co. (Shanghai, China). The shRNA sequence against BLACAT1 is 5'-AGGCUGGUUUCUGCCCUCAUCCUUU-3', and the control sequence is 5'-UUUCUCCGAACGUGUCACGUT T-3'. The transfections were performed by Lipofectamine 2000 (Invitrogen, NY, USA) according to the manufactures' instructions. Six hours after transfection, the culture medium was replaced with fresh DMEM.

RNA isolation and RT-qPCR. Total RNAs from human tissues and cultured cells were extracted with TRIzol ${ }^{\circledR}$ reagent (Takara Bio, Inc., Otsu, Japan) in a dilution of $1 \mathrm{ml}$ for each well of a six-well plate. The RNA quality and concentration were determined by collecting the absorbance using the Nanodrop 2000 spectrophotometer (Thermo Fisher Scientific, Inc., Beijing, China) at 260 and $280 \mathrm{~nm}$. Reverse transcription (RT) of first-strand cDNAs was conducted by PrimeScript RT Master Mix (Perfect Real Time; Takara Bio, Inc.) according to the manufacturer's protocol. All PCR reactions were performed in an ABI PRISM 7900 Real-Time system (Applied
Biosystems; Thermo Fisher Scientific, Inc.) with the SYBR ${ }^{\circledR}$ Premix Ex Taq $^{\mathrm{TM}}$ kit (Takara Bio, Inc). The thermocycling protocol was listed as follows: Initial denaturation at $95^{\circ} \mathrm{C}$ for $2 \mathrm{~min}$, followed by 35 repeats of the three-step cycling program consisting of $30 \mathrm{sec}$ at $95^{\circ} \mathrm{C}$ (denaturation), $1 \mathrm{~min}$ at $53^{\circ} \mathrm{C}$ (primer annealing) and $30 \mathrm{sec}$ at $72^{\circ} \mathrm{C}$ (elongation), followed by a final extension step for $10 \mathrm{~min}$ at $72^{\circ} \mathrm{C}$. The primer sequences used for qPCR are listed below: BLACAT1: Sense, 5'-GTCTCTGCCCTTTTGAGCCT-3' and antisense, 5'-GTG GCTGCAGTGTCATACCT-3'; GAPDH: Sense: 5'-GGGAAA CTGTGGCGTGAT-3' and antisense, 5'-GAGTGGGTGTCG CTGTTGA-3'. The housekeeping gene GAPDH was used as the internal control. Primers were purchased from Shenggong Co. (Shanghai, China). All quantitative data were normalized to GAPDH using the $2^{-\Delta \Delta \mathrm{Cq}}$ method (16).

Colony formation assay. ME180 and C33A cells were transfected with shNC or shBLACAT1 and cultivated in six-well plates at a density of 200 cells/well. After 2 weeks in $37^{\circ} \mathrm{C}$ incubator, the cell colonies that contained $>50$ cells were counted by staining with $0.5 \%$ crystal violet for $10 \mathrm{~min}$ and observation under a light microscope with a magnification of 200x (Nikon, Tokyo, Japan).

Cell cycle analysis. Prior to tests, ME180 and C33A cells were transfected with shRNAs with or without BLACAT1 knockdown for $72 \mathrm{~h}$. Next, cells were collected by low speed centrifuge $\left(840 \mathrm{~g}, 5 \mathrm{~min}, 4^{\circ} \mathrm{C}\right)$ and fixed by pre-cold ethanol $(70 \%)$ for $10 \mathrm{~min}$ on ice. The cells were washed and re-suspended in pre-cold PBS and incubated at $37^{\circ} \mathrm{C}$ for $30 \mathrm{~min}$ with $10 \mathrm{mg} / \mathrm{ml} \mathrm{RNase}$ and $1 \mathrm{mg} / \mathrm{ml}$ propidium iodide (PI) (Sigma-Aldrich, St Louis, MO, USA). The percentage of cells in each cell cycle phase was determined using the Cell Quest acquisition software (BD Biosciences, San Diego, CA, USA). Cell proliferation assay. Both ME180 and C33A cells were seeded in a 96-well plate at a concentration of $1 \times 10^{3} /$ well. After incubation for $24 \mathrm{~h}$, cells were transfected with shRNA against BLACAT1 or control shRNA. Cell proliferation was examined every day in the consecutive 5 days using a CellTiter 96 AQueous Non-Radioactive Cell Proliferation kit (Promega Corp., Madison, WI, USA) following the manufacturer's protocol. The cell proliferation rate was determined by measuring the absorbance at $490 \mathrm{~nm}$ using a microplate reader (Tecan, Männedorf, Switzerland).

Transwell assay. For cell migration assays, ME180 and C33A cells were trypsinized and collected by low-speed centrifugation $\left(840 \mathrm{~g}, 4^{\circ} \mathrm{C}\right.$, and $\left.5 \mathrm{~min}\right)$ with serum-free medium. A total of $1 \times 10^{4}$ cells $(\sim 150 \mu \mathrm{l})$ were spread into the upper chamber. The lower chamber was filled with $600 \mu 1$ medium containing $10 \%$ FBS. Afterwards, the plate was incubated in the $37^{\circ} \mathrm{C}$ incubator and the cells are allowed to grow freely. At $24 \mathrm{~h}$ post-seeding, the membrane was fixed with pre-cooled methanol and stained with crystal violet (1\%) for $5 \mathrm{~min}$ at room temperature. Cell migration was assessed by counting the cells that had migrated through the membrane. Five random fields were selected and images captured under a Nikon light microscope (Nikon) at a magnification of 100x. For cell invasion assays, the membrane was pre-coated with Matrigel (Corning Inc., Corning, NY, USA) for $6 \mathrm{~h}$ at $37^{\circ} \mathrm{C}$ incubator. 
Wound-healing assay. ME180 and C33A cells were transfected with shRNA against BLACAT1 or control shRNA, and were then cultured in DMEM in a six-well culture plate at a density of $5 \times 10^{5}$ cells/well and allowed to grow to a confluence of $90 \%$ overnight. The culture medium was replaced with serum-free DMEM. A line was scratched in the single cell layer using a $10 \mu \mathrm{l}$ pipette tip and the cells were then washed with PBS three times. Following incubation for $12 \mathrm{~h}$, images of the migrating cells were observed and images captured using a Nikon light microscope.

Western blot analysis. Protein expression in the process of EMT and cell cycle were evaluated by western blot analysis. Briefly, total proteins from ME180 and C33A cells were collected by lysis buffer (NP-40; Beyotime, Nantong, China) on ice and quantified using Bio-Rad protein assay reagent (Bio-Rad Laboratories, Inc., Hercules, CA, USA). Equal amounts of protein $(50 \mu \mathrm{g})$ were loaded onto $10 \%$ sodium dodecyl sulfate-polyacrylamide gel for electrophoresis and then transferred to a nitrocellulose membrane (NC, Millipore, MA, USA). The membrane was blocked for $1 \mathrm{~h}$ with $5 \%$ skimmed milk at room temperature and then incubated with primary antibodies overnight at $4{ }^{\circ} \mathrm{C}$. The primary antibodies against Cyclin B1 (sc-70898, 1:1,000), CDC25C (sc-327, 1:1,000), E-Cadherin (sc-71009, 1:1,000), $\mathrm{N}$-Cadherin (sc-53488, 1:1,000) and GAPDH (sc-32233, 1:1,000) were purchased from Santa Cruz Biotechnology, Inc. (Dallas, TX, USA). After washing with TBST for 4 times, the membrane was then incubated with secondary goat-anti-rabbit (sc-2004) or goat-anti-mouse (sc-2005) antibody (Santa Cruz Biotechnology, Inc.) for $1 \mathrm{~h}$ at $37^{\circ} \mathrm{C}$ with a dilution of $1: 1,000$. Finally, the proteins were quantified using ECL Prime Western Blotting Detection reagent (GE Healthcare, Parsippany, NJ, USA) and an ImageQuant LAS 4000 Mini Biomolecular Imager (GE Healthcare).

Statistically analysis. In vitro experiments were repeated at least three times in triplicate to yield reproducible results. All data were presented as the mean \pm standard deviation (SD). Student's t-test analysis was used for the comparison between two groups. Data were analyzed using Prism 6 (GraphPad Software Inc., San Diego, CA, USA). P $<0.05$ was considered to indicate a statistically significant difference

\section{Results}

Long noncoding RNA BLACAT1 is upregulated in human cervical cancer in vivo and in vitro. A total of 100 cervical cancer patients were involved and both their tumor tissues and adjacent non-cancerous tissues were subjected for RT-PCR analysis after dissection. As shown in Fig. 1A, the relative transcript level of BLACAT1 was significantly higher in most of the tumor tissues (99/100) as compared with their counterparts $\left.{ }^{* * *} \mathrm{P}<0.0001\right)$. Primary normal cervical epithelial cells (NCECs) were from non-cancerous cervical tissues and used as a control. It was shown that the relative transcript level of BLACAT1 was remarkably upregulated in all of the cervical cancer cell lines, of which the ME180 and C33A cells showed the highest expression of BLACAT1 (Fig. 1B). Therefore, these two cell lines were selected for subsequent analysis. Our data
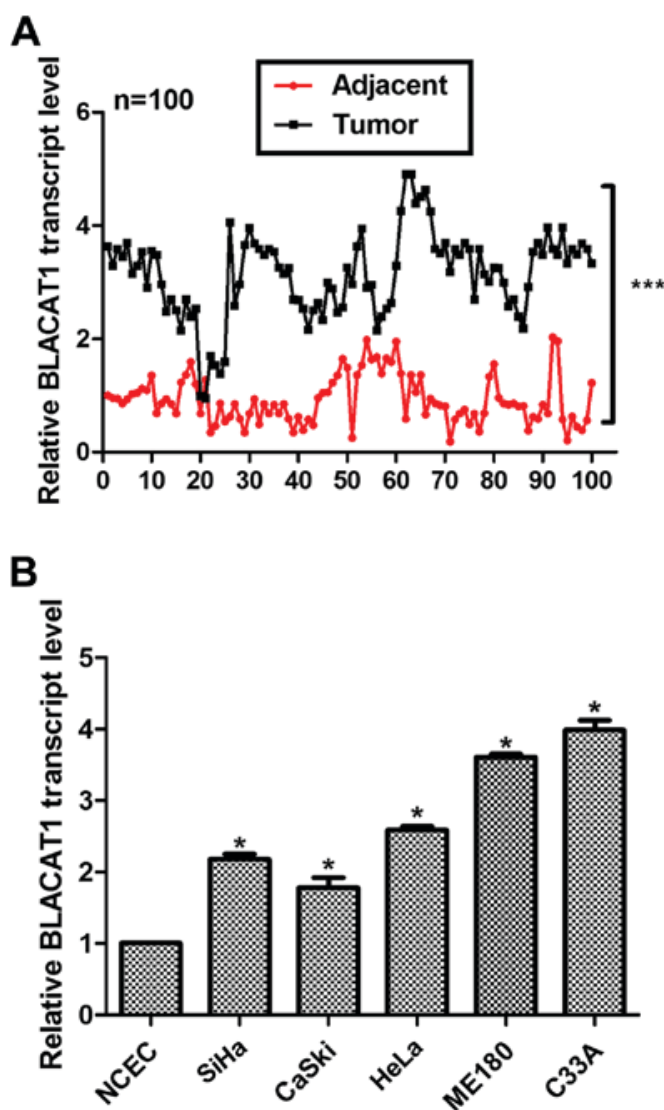

Figure 1. Long noncoding RNA BLACAT1 was upregulated in human cervical cancer in vivo and in vitro. (A) A total of 100 cervical cancer patients were included and RT-PCR analysis was used to assess the transcript level of BLACAT1. ${ }^{* * *} \mathrm{P}<0.0001$, vs. Adjacent. (B) RT-PCR analysis was performed to calculate the transcript level of BLACAT1 in NCEC control cells and five cervical cancer cell lines. ${ }^{*} \mathrm{P}<0.05$, vs. NCEC.

suggested that long noncoding RNA BLACAT1 was highly upregulated in human cervical cancer.

Knockdown of BLACAT1 inhibits cell proliferation in human cervical cancer in vitro. To explore the effects of BLACAT1 on human cervical cancer, specific shRNA were used to knock down the expression of BLACAT1 in ME180 and C33A cells. As shown in Fig. 2A, transfection of shBLACAT1 decreased the expression of BLACAT1 by more than $50 \%$ in both ME180 and C33A cells. Colony formation assay showed that approximate 250 colonies for control ME180 cells and 280 colonies for control C33A cells were formed; however, only 125 colonies for ME180 cells and 116 colonies for C33A cells were observed upon shBLACAT1 transfection (Fig. 2B and C). Moreover, knockdown of BLACAT1 in ME180 and C33A cells arrested cell cycle in G0/G1 phase, as evidenced by findings that the cell percentage in $\mathrm{G} 0 / \mathrm{G} 1$ phase was increased by $13 \%$ for ME180 cells and 15\% for C33A cells. Meanwhile, the cell percentage in $\mathrm{S}$ phase and G2/M phase was decreased accordingly for both cell lines (Fig. 2D-F). Cell proliferation was also determined in both cell lines upon shBLACAT1 transfection. There were no notable differences among three groups in the former three days in ME180 and C33A cells; however, on the fourth day, the cell proliferative rate was decreased by $18 \%$ for ME180 cells and 21\% for C33A cells. 
A

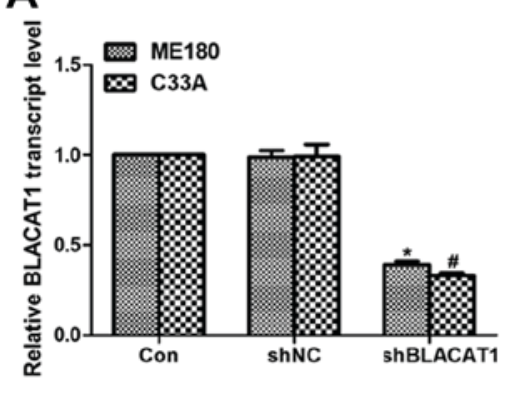

D

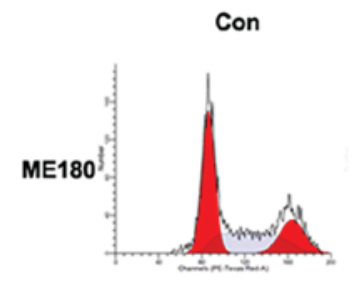

$\operatorname{shNC}$
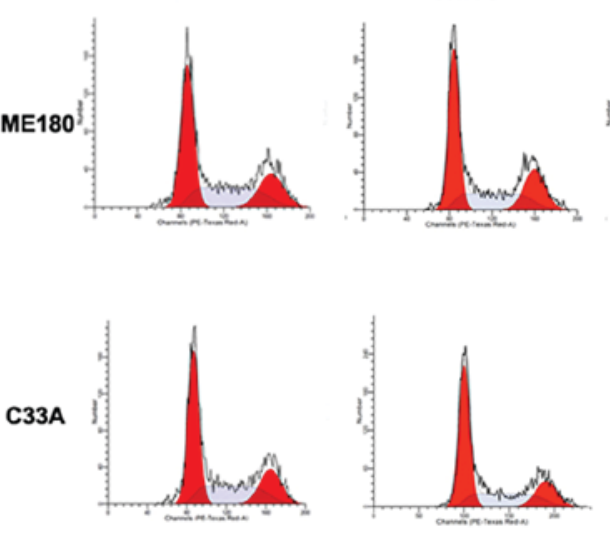

$\mathbf{F}$
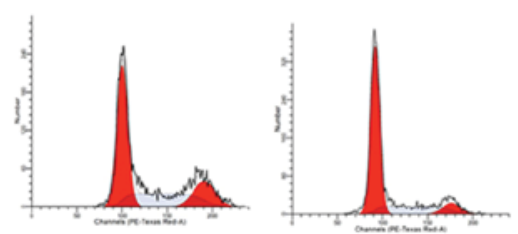

G
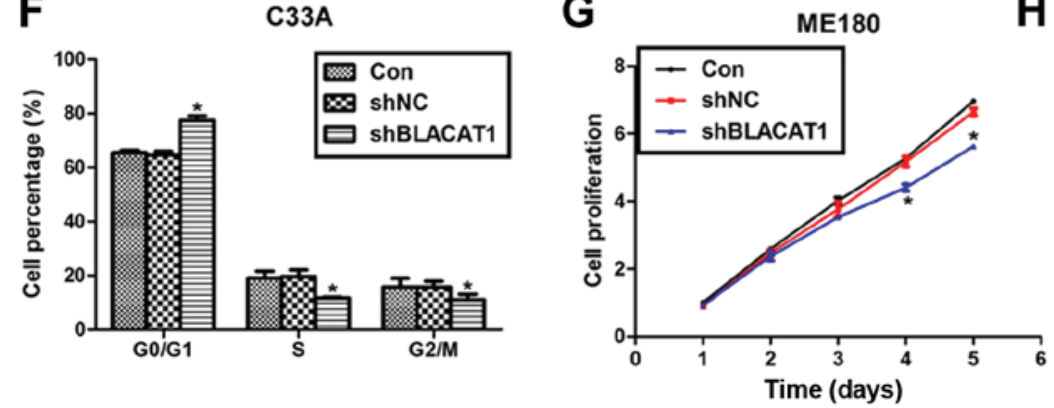

H
C

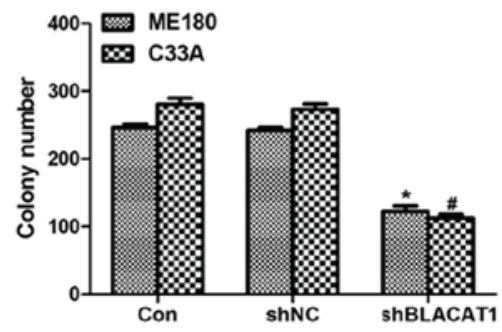

E

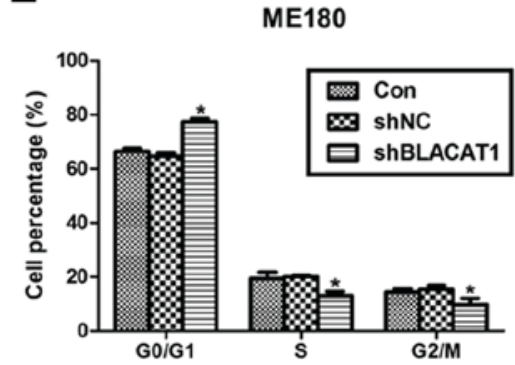

G0/G1
ShBLACAT1
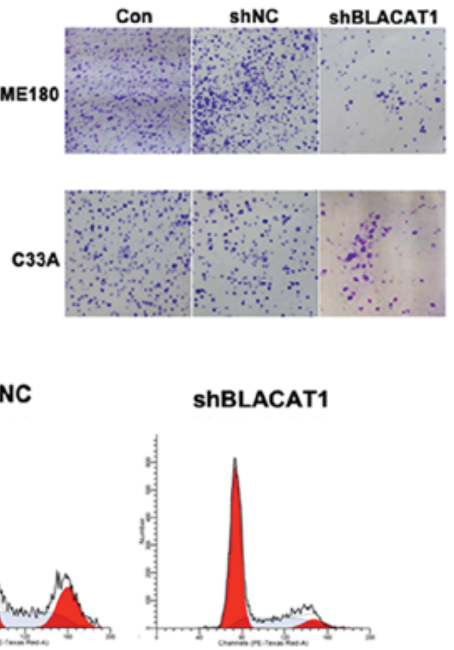

C33A

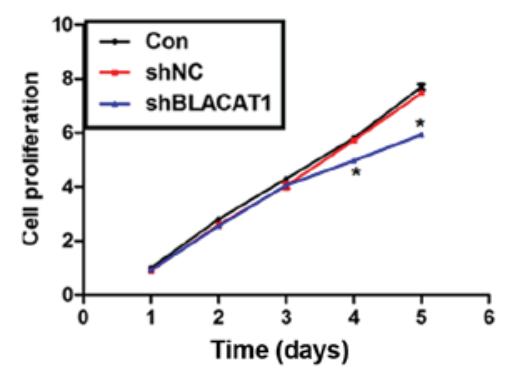

Figure 2. Knockdown of BLACAT1 inhibited cell proliferation in human cervical cancer in vitro. (A) RT-PCR analysis was performed to assess the expression of BLACAT1 in ME180 and C33A cells upon transfection of shBLACAT1. (B) Representative images of colony formation assays for ME180 and C33A cells (C) Colony formation assay was performed to assess the formed colonies of ME180 and C33A cells upon transfection of shBLACAT1. "P<0.05, vs. Con in ME180 cells. "P<0.05, vs. Con in C33A cells. (D) Representative images of cell cycle assays for ME180 and C33A cells. (E) Cell cycle analysis showed the cell percentage in each phase upon shBLACAT1 transfection in ME180 cells. (F) Cell cycle analysis showed the cell percentage in each phase upon shBLACAT1 transfection in C33A cells. (G) Cell proliferation assay was performed to reveal the cell proliferative rate of ME180 cells upon shBLACAT1 transfection in a continuous five days. $(\mathrm{H})$ Cell proliferation assay was performed to reveal the cell proliferative rate of C33A cells upon shBLACAT1 transfection in a continuous five days. "P<0.05, vs. Con.

The inhibitive effects were more obvious on the fifth day by the transfection of shBLACAT1 in both cell lines (Fig. 2G and $\mathrm{H})$. All of these data suggested that knockdown of BLACAT1 in ME180 and C33A cells inhibited cell proliferation in vitro.

Depletion of BLACAT1 suppresses cell metastasis in ME180 and C33A cells. Cell proliferation and cell metastasis are two main manifestations of various malignancy. Thus, the role of BLACAT1 in cell metastasis was also explored with Transwell assay and wound-healing analysis. It was shown that more than 200 ME180 cells and 240 C33A cells migrated through the membrane, and only 110 cells were observed after transfection of shBLACAT1 (Fig. 3A and B). Similarly, cell invasion was also inhibited by knockdown of BLACAT1 in ME180 and C33A cells. Approximate $100 \mathrm{ME} 180$ and C33A cells were observed to be retarded to invade through the membrane upon knockdown of BLACAT1 (Fig. 3A and C). Afterwards, wound-healing assay further demonstrated this observation again. Cell capacity to close the wound was inhibited by more than $50 \%$ after depletion of BLACAT1 in both cell lines (Fig. 3D and E). These results revealed that depletion of BLACAT1 inhibited cell metastasis in human cervical cancer in vitro.

Depletion of BLACAT1 in ME180 and C33A cells decreases cell cycle regulators and suppressed EMT process. EMT is associated with tumorigenesis. Therefore, cell cycle regulators cyclin $\mathrm{B} 1$ and $\mathrm{CDC} 25 \mathrm{C}$, and EMT markers including mesenchymal N-cadherin and epithelial E-cadherin, were detected in ME180 and C33A cells with shBLACAT1 transfection using western blot analysis. As presented in Fig. 4, 

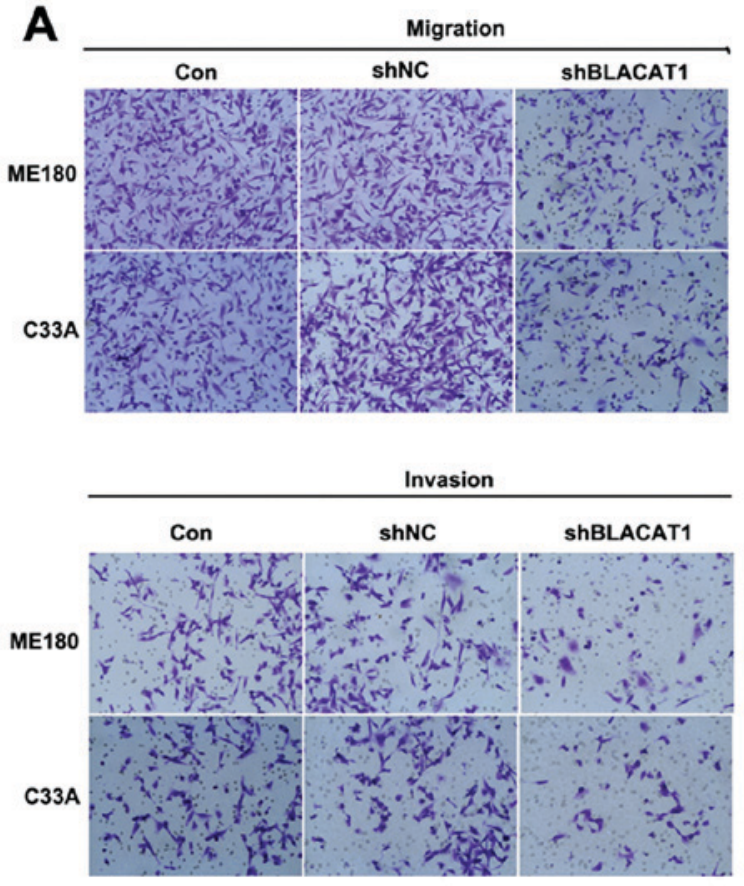

B

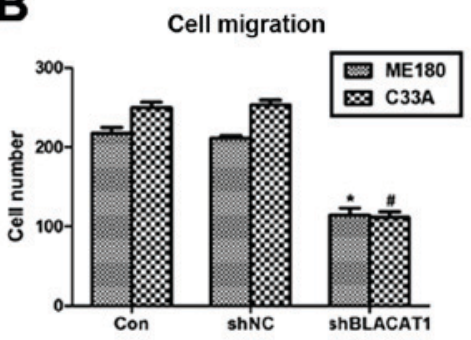

D ME180

$\mathrm{Oh}$

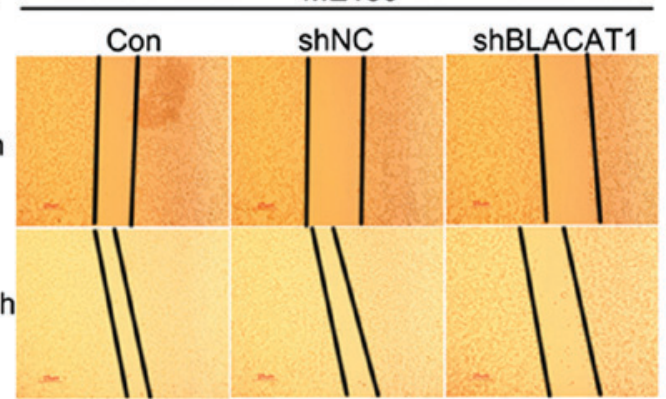

C33A

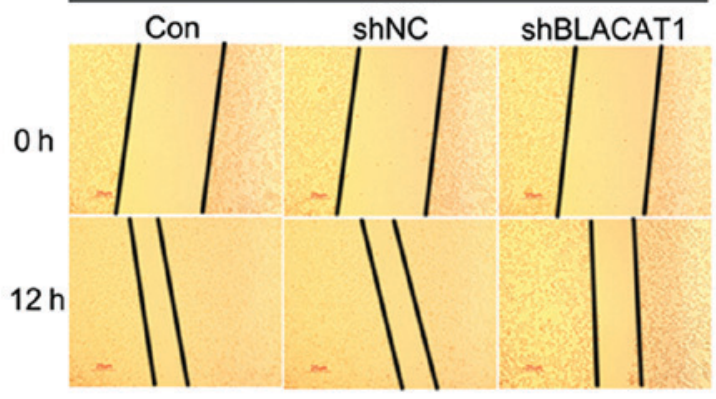

C

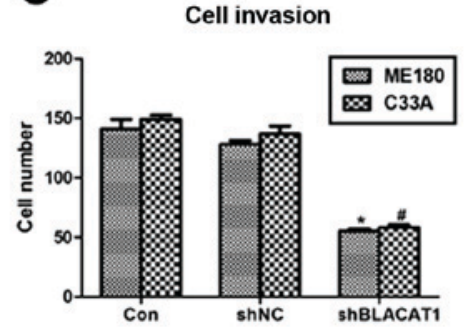

E

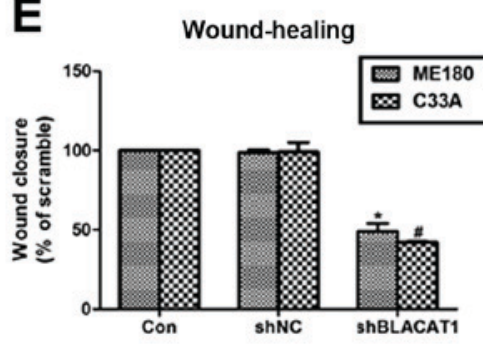

Figure 3. Depletion of BLACAT1 suppressed cell metastasis in ME180 and C33A cells. (A) Representative images of Transwell assays were presented after treatment of shBLACAT1 on ME180 and C33A cells. (B) Quantification of cell migration assays showed the cell number on the lower surface of membrane upon shBLACAT1 transfection in ME180 and C33A cells. (C) Quantification of cell invasion assays showed the cell number on the lower surface of membrane upon shBLACAT1 transfection in ME180 and C33A cells. ${ }^{*} \mathrm{P}<0.05$, vs. Con in ME180 cells. ${ }^{\prime \prime} \mathrm{P}<0.05$, vs. Con in C33A cells. (D) Representative images of wound-healing assays were presented after 0 or $12 \mathrm{~h}$ treatment of shBLACAT1 on ME180 and C33A cells. (E) Quantification of wound-healing assays showed the cell capacity to migrate and invade upon shBLACAT1 transfection in ME180 and C33A cells. ${ }^{*} \mathrm{P}<0.05$, vs. Con in ME180 cells. ${ }^{*} \mathrm{P}<0.05$, vs. Con in C33A cells.

after BLACAT1 was knocked down by specific shRNAs in cervical cancer ME180 and C33A cells, the protein levels of cyclin $\mathrm{B} 1, \mathrm{CDC} 25 \mathrm{C}$ and $\mathrm{N}$-cadherin were decreased, while the expression of E-cadherin was increased. The results of the present study were consistent with the above functional observations, reinforcing that knockdown of BLACAT1 inhibited cell proliferation and metastasis.

\section{Discussion}

Cervical cancer remains one of the most common gynecological malignancies (1). In spite of traditional treatments consisting of surgery, chemotherapy and radiotherapy the long-term prognosis for patients with cervical cancer remains poor due to metastatic or recurrent property (2). In view that uncontrolled cell growth and metastasis are common features of cervical cancer (4), identification of novel factors associated with cervical cancer cell proliferation, migration and invasion would aid in developing novel effective therapies.

Recent advances have implicated IncRNAs in the development and progression of cervical cancer. BLACAT1 is a newly

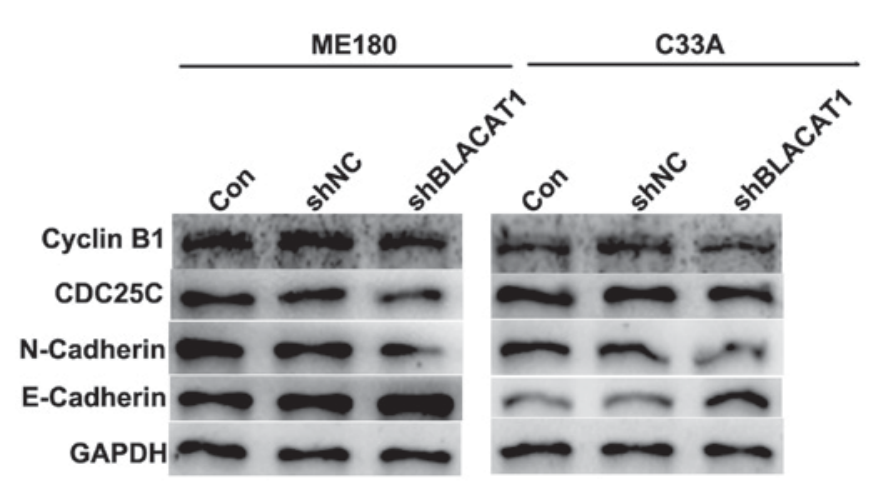

Figure 4. Depletion of BLACAT1 in ME180 and C33A cells decreased cell cycle markers and suppressed EMT process. Western blot analysis revealed that the protein levels of Cyclin B1, CDC25C, N-Cadherin, E-Cadherin and GAPDH altered upon transfection of shBLACAT1 in ME180 and C33A cells.

identified lncRNA that was initially characterized in bladder cancers (13), and later found to be associated with prognosis of gastric cancer (14) and colorectal cancer (15). The present 
study was the first one to uncover the expression and biological character of BLACAT1 in cervical cancer.

Our data have shown that the relative expression of BLACAT1 in cervical cancer tissues were significantly upregulated as compared with the adjacent non-cancerous tissues. This expression profile was consistent with previous studies $(14,15)$. Moreover, with the use of a specific shRNA against BLACAT1, it was further found that depletion of BLACAT1 inhibited cell proliferation in cervical cancer ME180 and C33A cells. Cell cycle deregulation is a hallmark of tumorigenesis and associated closely with aberrant cell growth (17). Cell growth primarily depends on cell cycle progression in mitosis and is basically regulated by cell cycle regulators, including cyclins and cyclin-dependent kinases (CDKs) $(18,19)$. Our western blot analysis revealed that knockdown of BLACAT1 decreased the protein levels of key cell cycle regulators Cyclin B1 and CDC25C, supporting the cell proliferation inhibition by BLACAT1 depletion. The accumulation of cells in G0/G1 phase with less cells in $\mathrm{S}$ and $\mathrm{G} 2 / \mathrm{M}$ phases also supported that the proliferation was inhibited after knockdown of BLACAT1 in ME180 and C33A cells. Interestingly, knockdown of BLACAT1 also slowed down wound recovery, migration and invasion capacities. Epithelial to mesenchymal transition is a hallmark of tumor distant metastasis (20). Decrease of epithelial markers and increase of mesenchymal markers were always accompanied with cancer malignancies. Our western blot analysis also revealed that epithelial property (E-cadherin) was over mesenchymal property (N-cadherin) after BLACAT1 knockdown, which significantly reinforced the metastasis-inhibition effects by BLACAT1 depletion. Therefore, our data have identified BLACAT1 as a critical mediator of cell proliferation and metastasis in cervical cancer.

Mechanisms of how BLACAT1 functions in cervical cancer remain mysterious to date. Available literature has shown that BLACAT1 exerts a functional role in bladder cancer by recruiting and binding to polycomb repressive complex 2 (PRC2) (13). A more recent study also reported that BLACAT1 regulated cell proliferation by epigenetically silencing of p15 (15). The two available studies suggested that regulation of proteins, either via physical interaction or epigenetic regulation, might underlie the functional basis of BLACAT1. However, more work needs to be done. We will further work to reveal these mechanistic networks that contributes to the biological character of BLACAT1 in cervical cancer and we will begin with the key regulator of PI3K/AKT, ERK1/2 AND $\mathrm{NF}-\kappa \mathrm{B}$ signaling pathway and examine the expression of p-AKT and p-ERK.

In all, the present study have identified a novel lncRNA, BLACAT1, as a critical mediator of cell proliferation and metastasis in cervical cancer. Our results have shown that BLACAT1 was highly expressed in cervical cancer. Depletion of BLACAT1 negatively affected cell proliferation, migration and invasion. Previous reports mainly focused on the clinical outcome of BLACAT1 in human cancers with its involvement in cancer cell aggressiveness largely unknown. Our data may represent the first one to provide experimental data supporting the pro-oncogenic property of BLACAT1 in cervical cancer. The conclusion drawn from the present study may aid in developing novel therapeutic strategies against cervical cancer in clinic.

\section{References}

1. Arbyn M, Castellsagué X, de Sanjosé S, Bruni L, Saraiya M, Bray F and Ferlay J: Worldwide burden of cervical cancer in 2008. Ann. Oncol 22: 2675-2686, 2011.

2. Yeung TL, Leung CS, Yip KP, Au Yeung CL, Wong ST and Mok SC: Cellular and molecular processes in ovarian cancer metastasis. A Review in the Theme: Cell and molecular processes in cancer metastasis. Am J Physiol Cell Physiol 309: C444-C456, 2015.

3. Zhang Y, Gao H, Gao X, Huang S, Wu K, Yu X, Yuan K and Zeng T: Elevated expression of Kin17 in cervical cancer and its association with cancer cell proliferation and invasion. Int $\mathrm{J}$ Gynecol Cancer 27: 628-633, 2017.

4. Zhou N, Fei D, Zong S, Zhang M and Yue Y: MicroRNA-138 inhibits proliferation, migration and invasion through targeting hTERT in cervical cancer. Oncol Lett 12: 3633-3639, 2016.

5. de Hoon M, Shin JW and Carninci P: Paradigm shifts in genomics through the FANTOM projects. Mamm Genome 26: 391-402, 2015.

6. Pennisi E: Genomics. ENCODE project writes eulogy for junk DNA. Science 337: 1159, 1161, 2012

7. Guo X and Hua Y: CCAT1: An oncogenic long noncoding RNA in human cancers. J Cancer Res Clin Oncol 143: 555-562, 2017.

8. Martin L and Chang HY: Uncovering the role of genomic 'dark matter' in human disease. J Clin Invest 122: 1589-1595, 2012.

9. Han P, Li W, Lin CH, Yang J, Shang C, Nuernberg ST, Jin KK, $\mathrm{Xu} \mathrm{W}$, Lin CY, Lin CJ, et al: A long noncoding RNA protects the heart from pathological hypertrophy. Nature 514: 102-106, 2014.

10. Hu Y, Sun X, Mao C, Guo G, Ye S, Xu J, Zou R, Chen J, Wang L, Duan P and Xue X: Upregulation of long noncoding RNA TUG1 promotes cervical cancer cell proliferation and migration. Cancer Med 6: 471-482, 2017.

11. Han J, Zhou W, Jia M, Wen J, Jiang J, Shi J, Zhang K, Ma H, Liu J, Ren J, et al: Expression quantitative trait loci in long non-coding RNA PAX8-AS1 are associated with decreased risk of cervical cancer. Mol Genet Genomics 291: 1743-1748, 2016.

12. Wu L, Jin L, Zhang W and Zhang L: Roles of long non-coding RNA CCAT2 in cervical cancer cell growth and apoptosis. Med Sci Monit 22: 875-879, 2016.

13. He W, Cai Q, Sun F, Zhong G, Wang P, Liu H, Luo J, Yu H, Huang $J$ and Lin T: Linc-UBC1 physically associates with polycomb repressive complex 2 (PRC2) and acts as a negative prognostic factor for lymph node metastasis and survival in bladder cancer. Biochim Biophys Acta 1832: 1528-1537, 2013.

14. Hu Y, Pan J, Wang Y, Li L and Huang Y: Long noncoding RNA linc-UBC1 is negative prognostic factor and exhibits tumor pro-oncogenic activity in gastric cancer. Int J Clin Exp Pathol 8: 594-600, 2015.

15. Su J, Zhang E, Han L, Yin D, Liu Z, He X, Zhang Y, Lin F, Lin Q, Mao P, et al: Long noncoding RNA BLACAT1 indicates a poor prognosis of colorectal cancer and affects cell proliferation by epigenetically silencing of p15. Cell Death Dis 8: e2665, 2017.

16. Livak KJ and Schmittgen TD: Analysis of relative gene expression data using real-time quantitative PCR and the 2(-Delta Delta C(T)) method. Methods 25: 402-408, 2001.

17. Sherr CJ: Cancer cell cycles. Science 274: 1672-1677, 1996.

18. Benson C, Kaye S, Workman P, Garrett M, Walton M and de Bono J: Clinical anticancer drug development: Targeting the cyclin-dependent kinases. Br J Cancer 92: 7-12, 2005.

19. Coudreuse D and Nurse P: Driving the cell cycle with a minimal CDK control network. Nature 468: 1074-1079, 2010.

20. Nieto MA, Huang RY, Jackson RA and Thiery JP: EMT: 2016. Cell 166: 21-45, 2016.

This work is licensed under a Creative Commons Attribution-NonCommercial-NoDerivatives 4.0 International (CC BY-NC-ND 4.0) License. 